\section{(1)}

CrossMark

\title{
Clofazimine does not lead to significant QT interval prolongation: a multicentre study
}

\author{
To the Editor:
}

We read with great interest the article by PonTALI et al. [1], entitled "Cardiac safety of bedaquiline: a systematic and critical analysis of the evidence". In response to this paper and the debate on potential QTc prolongation in clofazimine- and bedaquiline-containing multidrug-resistant tuberculosis (MDR-TB) treatment regimens $[2,3]$, we assessed QTc prolongation during clofazimine use in treatment of disease caused by nontuberculous mycobacteria (NTM). This is important as NTM disease treatment typically includes multiple potential QTc-prolonging antibiotics, including macrolides, fluoroquinolones and, increasingly, clofazimine [4].

In a mixed prospective and retrospective study promoted through the NTM-NET (NTM Network European Trials Group) website (www.ntm-net.org; study \#7), we measured QTc intervals in patients on clofazimine-based regimens for NTM disease. We only enrolled patients who had at least one ECG at the start of therapy (or in the 3 weeks before or after the start) and one ECG after at least 3 months of clofazimine use, to ensure steady state was reached [5]. Patients with pre-existing QTc prolongation and those simultaneously using bedaquiline were excluded from the study.

Enrolment of eligible patients was started in October 2012 and continued until September 2017. We applied American Heart Association standards and the Bazett formula to calculate QTc. Severe QTc prolongation, necessitating interruption of drugs, was defined as a QTc interval $\geqslant 500 \mathrm{~ms}$ [6]. Electrolyte measurements were available for some of the patients.

We enrolled 18 patients (11 prospective, seven retrospective): 10 (56\%) men and eight women, with a mean age of $49.7 \pm 21.1$ years and a mean QTc at baseline of $404.4 \pm 28.2 \mathrm{~ms} .15$ patients had pulmonary NTM disease, three patients had extrapulmonary disease; causative species are presented in figure 1a. All patients were treated with at least two QTc-prolonging antimycobacterial drugs (clofazimine and macrolide). 13 (72\%) patients used at least one (up to five) other potentially QTc-prolonging drugs, e.g. proton pump inhibitors in nine patients and anti-emetics in six. $33 \%$ of the patients had one follow-up ECG, 28\% had two follow-up ECGs, 17\% had three follow-up ECGs and 22\% had five or more follow-up ECGs. The last available follow-up ECG was made after a mean of $213.2 \pm 168.1$ days after start of clofazimine therapy.

For Mycobacterium avium complex (MAC) disease (M. avium, Mycobacterium intracellulare, Mycobacterium chimaera and untypeable MAC), a clofazimine-ethambutol-macrolide regimen was given to seven $(77.8 \%)$ out of the nine patients. Four of these patients also used a rifamycin and amikacin. Two patients had a clofazimine-rifamycin-macrolide regimen. The core treatment regimen for six Mycobacterium abscessus patients consisted of clofazimine-macrolide-amikacin-imipenem (or cefoxitin); four received additional tigecycline. One patient, with Mycobacterium tilburgii disease, had a clofazimine-macrolide-rifabutin-protionamide regimen.

In our cohort, the administration of clofazimine did not lead to significant QTc prolongation. The maximum change in QTc between baseline and follow-up ECG was a mean of $39.2 \pm 24.5$ ms (figure 1b), which falls within the changes of 50-65 ms considered normal variation by circadian rhythm [6]. All QTc interval lengths measured during treatment are shown in figure 1c. The patterns of intra-patient

@ERSpublications

Clofazimine does not lead to significant QT interval prolongation in the treatment of nontuberculous mycobacteria http://ow.ly/sXC330lIbI8

Cite this article as: Zweijpfenning $\mathrm{SMH}$, van Groningen $\mathrm{H}$, van Ingen J, et al. Clofazimine does not lead to significant QT interval prolongation: a multicentre study. Eur Respir J 2018; 52: 1801386 [https://doi. org/10.1183/13993003.01386-2018]. 
a)

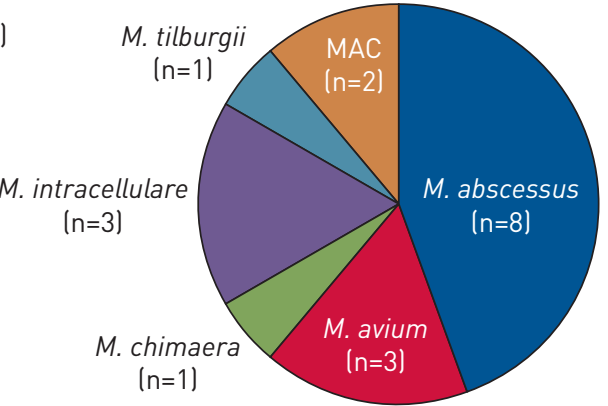

$(n=1)$ c)

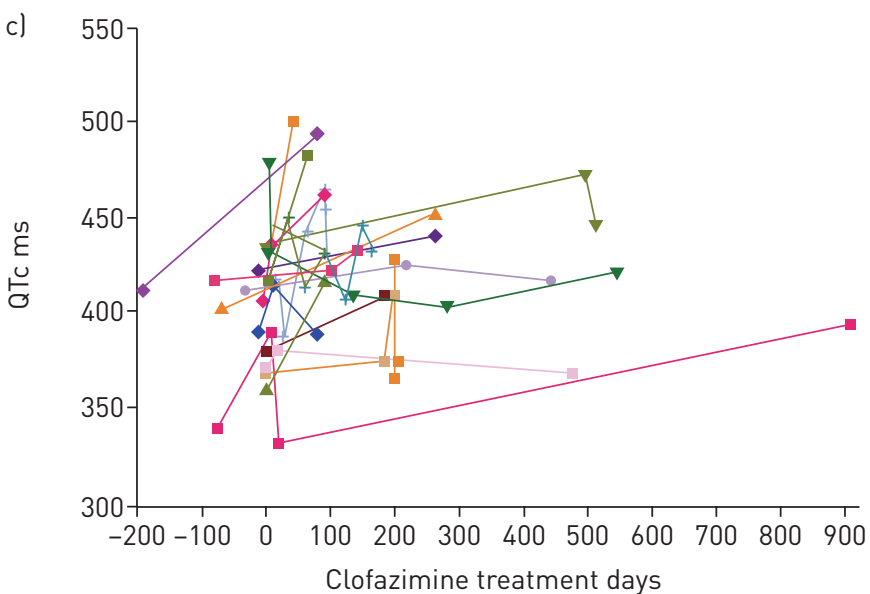

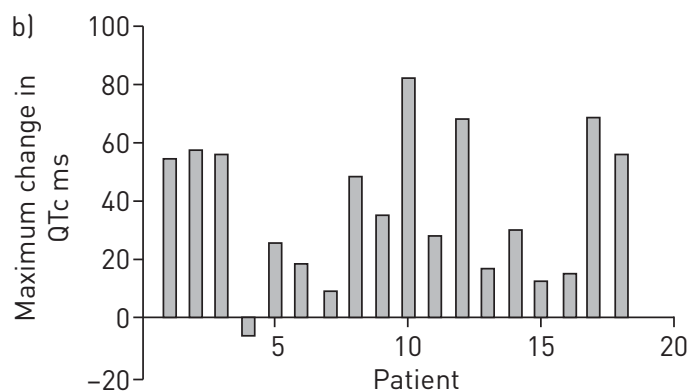

Patient 1

-- Patient 2

^- Patient 3

- Patient 4

- Patient 5

- Patient 6

- Patient 7

- Patient 8

$\rightarrow$ Patient 9

- Patient 10

- Patient 11

+ Patient 12

+ Patient 13

+ Patient 14

$\rightarrow$ Patient 15

- Patient 16

- Patient 17.1

- Patient 17.2

- Patient 18

FIGURE 1 a) Causative nontuberculous mycobacteria species for the 18 patients included in this study. $M$. tilburgii: Mycobacterium tilburgii; MAC: untypeable Mycobacterium avium complex; $M$. intracellulare: Mycobacterium intracellulare; M. abscessus: Mycobacterium abscessus; M. chimaera: Mycobacterium chimaera; M. avium: Mycobacterium avium. b) Maximum change in QTc from baseline during follow-up, per patient. c) QTc intervals measured during follow-up. Patient 17 was treated twice, hence the labels 17.1 and 17.2.

fluctuation also suggest this may be mere circadian rhythm. One patient developed a QTc interval of $501 \mathrm{~ms}$ after 6 weeks of clofazimine-macrolide-amikacin-tigecycline-imipenem treatment for NTM disease, with concomitant itraconazole and ondansetron which can cause QTc prolongation. After treatment cessation and re-introduction of antimycobacterial drugs, but not azoles and ondansetron, this patient no longer showed QTc interval prolongation.

Studies in tuberculosis patients receiving bedaquiline and clofazimine have hinted at clofazimine as a cause of QTc prolongation [2]. Two recent studies have addressed the safety of clofazimine-based treatment regimens for NTM disease, including some QTc interval data. In one study, 40 patients had ECGs but none showed QTc intervals $\geqslant 500 \mathrm{~ms}$ (highest $473 \mathrm{~ms}$ ) [7]. The second study included 35 patients using clofazimine for MDR-TB or NTM disease; one patient had to stop clofazimine-moxifloxacin-macrolide treatment because of QTc interval prolongation $(550 \mathrm{~ms})$ and one patient experienced cardiac arrest in the absence of QTc interval prolongation $(445 \mathrm{~ms}$ ) [8]. Furthermore, in a study in tuberculosis patients, clofazimine use was safe, but a trend towards more frequent deaths was observed in this group [9]. Such findings could not be replicated in our smaller study.

QTc interval prolongation observed with clofazimine and bedaquiline use could have been primarily due to bedaquiline use, or specifically to the bedaquiline-clofazimine combination [2, 3, 10]. This warrants future prospective studies, as this combination is likely to gain importance in mycobacterial disease treatment [5].

The main limitation of our study is the fact that follow-up was not standardised, due to its observational character. Whether QTc prolongation is dependent on clofazimine's slow accumulation in tissues is not known [5]. Long-term follow-up with frequent ECGs in larger cohorts is needed to settle this issue. Also, we applied a definition of $>500 \mathrm{~ms}$ as QTc prolongation, although the US Food and Drug Administration guide for industry suggests that drug-induced increases $>60 \mathrm{~ms}$ (observed in three of our patients) should be regarded as QTc prolongation [6]. 
In summary, clofazimine did not cause significant QTc interval prolongation in this small cohort of patients treated for NTM disease. In patients with an increasing QTc interval, this could not be related specifically to clofazimine use; concomitant medication should also be used with caution. Critical review of QTc-prolonging drugs and follow-up of electrolyte levels and ECGs are still advised in patients starting clofazimine-based NTM disease treatment.

Sanne M.H. Zweijpfenning ${ }^{1}$, Hester van Groningen ${ }^{1}$, Jakko van Ingen $\odot^{2}$, Cecile Magis-Escurra ${ }^{1}$, Martin J. Boeree ${ }^{1}$, Dirk Wagner, Hannelore $\mathrm{Bax}^{4}$, Claire Andrejak, Reinier Wener, Ananna Rahman, Heinke Kunst ${ }^{8}$, Natalie Lorent ${ }^{9}$, Marc A. Brouwer ${ }^{10}$ and Wouter Hoefsloot $\oplus^{1}$, on behalf of NTM-NET

${ }^{1}$ Dept of Pulmonary Diseases, Radboud University Medical Center, Nijmegen, The Netherlands. ${ }^{2}$ Dept of Medical Microbiology, Radboud University Medical Center, Nijmegen, The Netherlands. ${ }^{3}$ Dept of Infectious Diseases, University Medical Center Freiburg, Freiburg, Germany. ${ }^{4}$ Dept of Internal Medicine, Erasmus Medical Center, Rotterdam, The Netherlands. ${ }^{5}$ Dept of Pulmonary Diseases, Centre Hospitalier Universitaire Amiens, Amiens, France. ${ }^{6}$ Dept of Pulmonary Diseases, Utrecht University Medical Center, Utrecht, The Netherlands. ${ }^{7}$ Dept of Respiratory Medicine, Bart's Hospital, London, UK. ${ }^{8}$ Queen Mary University of London, Barts and The London School of Medicine and Dentistry, London, UK. ${ }^{9}$ Dept of Pulmonary Diseases, University Hospitals Leuven, Leuven, Belgium. ${ }^{10}$ Dept of Cardiology, Radboud University Medical Center, Nijmegen, The Netherlands.

Correspondence: Sanne M.H. Zweijpfenning, Radboud University Medical Center Nijmegen, Dept of Pulmonary Diseases, Internal postal code 614, Postbus 9101, 6500HB Nijmegen, The Netherlands.

E-mail: Sanne.Zweijpfenning@radboudumc.nl

Received: April 042018 | Accepted after revision: Aug 222018

Conflict of interest: S.M.H. Zweijpfenning reports grants and non-financial support for a clinical trial from Novartis and Insmed, outside the submitted work. H. van Groningen has nothing to disclose. J. van Ingen has nothing to disclose. C. Magis-Escurra has nothing to disclose. M.J. Boeree has nothing to disclose. D. Wagner has nothing to disclose. H. Bax nothing to disclose. C. Andrejak has nothing to disclose. R. Wener has nothing to disclose. A. Rahman has nothing to disclose. H. Kunst has nothing to disclose. N. Lorent has nothing to disclose. M.A. Brouwer has nothing to disclose. W. Hoefsloot reports grants from Insmed Corp, during the conduct of the study.

\section{References}

1 Pontali E, Sotgiu G, Tiberi S, et al. Cardiac safety of bedaquiline: a systematic and critical analysis of the evidence. Eur Respir J 2017; 50: 1701462.

2 Wallis RS. Cardiac safety of extensively drug-resistant tuberculosis regimens including bedaquiline, delamanid and clofazimine. Eur Respir J 2016; 48: 1526-1527.

3 Tadolini $\mathrm{M}$, Lingtsang $\mathrm{RD}$, Tiberi $\mathrm{S}$, et al. Cardiac safety of extensively drug-resistant tuberculosis regimens including bedaquiline, delamanid and clofazimine. Eur Respir J 2016; 48: 1527-1529.

4 van Ingen J, Ferro BE, Hoefsloot W, et al. Drug treatment of pulmonary nontuberculous mycobacterial disease in HIV-negative patients: the evidence. Expert Rev Anti Infect Ther 2013; 11: 1065-1077.

5 Cholo MC, Mothiba MT, Fourie B, et al. Mechanisms of action and therapeutic efficacies of the lipophilic antimycobacterial agents clofazimine and bedaquiline. J Antimicrob Chemother 2017; 72: 338-353.

6 Rautaharju PM, Surawicz B, Gettes LS, et al. AHA/ACCF/HRS recommendations for the standardization and interpretation of the electrocardiogram. Part IV: the ST segment, T and U waves, and the QT interval. A scientific statement from the American Heart Association Electrocardiography and Arrhythmias Committee, Council on Clinical Cardiology; the American College of Cardiology Foundation; and the Heart Rhythm Society. Endorsed by the International Society for Computerized Electrocardiology. J Am Coll Cardiol 2009; 53: 982-991.

7 Martiniano SL, Wagner BD, Levin A, et al. Safety and effectiveness of clofazimine for primary and refractory nontuberculous mycobacterial infection. Chest 2017; 152: 800-809.

8 Yoon HY, Jo KW, Nam GB, et al. Clinical significance of QT-prolonging drug use in patients with MDR-TB or NTM disease. Int J Tuberc Lung Dis 2017; 21: 996-1001.

9 Dalcolmo M, Gayoso R, Sotgiu G, et al. Effectiveness and safety of clofazimine in multidrug-resistant tuberculosis: a nationwide report from Brazil. Eur Respir J 2017; 49: 1602445.

10 Dannemann B, Bakare N, De Marez T, et al. QTcF prolongation in a phase II trial of TMC207 plus background regimen as treatment for multidrug-resistant tuberculosis: effect of co-administration with clofazimine. Presented at: 52nd Interscience Conference on Antimicrobial Agents and Chemotherapy, San Francisco, CA, USA; September 9-12, 2012. Poster A-1259. 